\title{
Lidil
}

Revue de linguistique et de didactique des langues

$43 \mid 2011$

Le rapport au savoir dans les discours professionnels

\section{Se reconnaitre, être reconnu comme professionnel}

To recognize oneself, to be recognized as a profesional

\section{Michèle Lusetti}

\section{(2) OpenEdition}

Journals

Édition électronique

URL : http://journals.openedition.org/lidil/3117

DOI : 10.4000/lidil.3117

ISSN : 1960-6052

\section{Éditeur}

UGA Éditions/Université Grenoble Alpes

\section{Édition imprimée}

Date de publication : 30 mai 2011

Pagination : 117-131

ISBN : 978-2-84310-201-1

ISSN : $1146-6480$

Référence électronique

Michèle Lusetti, « Se reconnaitre, être reconnu comme professionnel », Lidil [En ligne], 43 | 2011, mis en ligne le 30 novembre 2012, consulté le 23 avril 2019. URL : http://journals.openedition.org/ lidil/3117; DOI : 10.4000/lidil.3117 


\title{
Se reconnaitre, être reconnu comme professionnel
}

\author{
Michèle Lusetti*
}

\begin{abstract}
RÉSUMÉ
Récent dans la formation des enseignants, le mémoire professionnel reste un genre aux contours fluctuants. Il est envisagé ici comme un écrit de formation réflexif qui permet à son auteur de se reconnaitre et d'être reconnu comme nouveau professionnel. À titre heuristique, l'étude porte sur l'analyse d'un mémoire professionnel de professeur des écoles évalué positivement. Avec les outils de l'analyse des discours et de la linguistique énonciative, l'auteure cherche à voir quels savoirs s'y construisent et comment ils s'y énoncent à partir de quatre postes d'observation principaux concernant la construction de diverses identités énonciatives, le dialogue avec les savoirs d'autrui dans le travail de citation, la présentation de savoirs issus de l'expérience et le bilan final d'un parcours de savoir. L'étude conduit à repérer un faisceau de propriétés qui pourrait caractériser un mémoire professionnel.
\end{abstract}

\section{ABSTRACT}

Recently introduced into teacher training in France, the professional dissertation still remains an ill-defined genre. It is considered in this paper as a written reflexive training tool allowing its author to recognize him/herself and to be recognized as a new member of the profession. This heuristic study focusses on the analysis of a positively assessed professional dissertation written by a primary school teacher. Using the theoretical tools of discourse analysis and enunciative linguistics, the author attempts in this paper to discover what kinds of knowledge are being built and how they are expressed verbally. Four main areas are observed: the construction of various enunciative identities, the dialogue with the knowledge of others through referencing, the presentation of empirical knowledge and the final outcome of a knowledge building process. The study leads to the identification of the features that could characterise a professional dissertation.

* Université Claude Bernard-Lyon 1, IUFM

ICAR, UMR 5191, Université Lumière-Lyon 2. 
Dans de nombreux secteurs, le mémoire professionnel est utilisé comme modalité de formation sous le paradigme de l'écriture considérée, notamment dans la mouvance des travaux de Goody, comme outil de pensée, visant à former un "praticien réflexif» selon l'expression inaugurée par Schön (1994). Mis en place en 1991 dans la formation des enseignants, il est un genre récent. Sans tradition rhétorique universitaire, il reste un genre aux contours fluctuants selon les cultures régionales, les disciplines et les partis pris idéologiques.

La littérature parue sur le sujet ${ }^{1} \mathrm{~s}$ 'avère être généralement un discours du déficit - sinon de la déploration - qui repère essentiellement les faiblesses des auteurs. Souvent on y constate les dérives de propositions qui restent technicistes, morcelées et parfois contradictoires, selon des modèles dont on ne voit pas que des cadres praxéologiques différents en changent les caractéristiques et les finalités.

À côté de cette littérature du manque, il se peut que les choix diffèrent : de même que parfois le sociologue s'inscrit dans la logique d'une sociologie participative ou que le linguiste s'empare d'un devoir d'élève en lecteur coopérant cherchant la cohérence plutôt qu'en correcteur traquant l'erreur (Garcia-Debanc, 1994), il arrive que soient analysées les qualités des meilleurs mémoires ou qu'ils soient, à travers des entretiens avec leurs auteurs, étudiés dans une démarche compréhensive qui permet d'accéder à la singularité du sujet.

À l'heure où la formation des enseignants est engagée dans de nouvelles voies, c'est dans cette perspective que nous nous situerons de façon à cerner comment un mémoire évalué positivement peut contribuer à la formation d'un professeur des écoles débutant. Nous chercherons à voir quels savoirs spécifiques s'y construisent et comment ils s'énoncent à partir de quatre postes d'observation principaux concernant la construction de diverses identités énonciatives, le dialogue avec les savoirs d'autrui dans le travail de citation, la présentation de savoirs issus de l'expérience et le bilan final d'un parcours de savoir.

1. Pour une synthèse voir notamment Cros (1998), Gomez (2001), Crinon (2003), Gonnin-Bolo (2004) et les revues Enjeux ( ${ }^{\circ}$ 54, 2002), Spirale $\left(\mathrm{n}^{\circ} 29,2002\right)$, Pratiques ( $\left.\mathrm{n}^{\circ} 121-122,2004\right)$, Lidil $\left(\mathrm{n}^{\circ} 34,2006\right)$ et Pratiques $\left(n^{\circ} 143-144,2009\right)$. 


\section{Choix théoriques et méthodologiques}

Selon Ricœur (2004), il apparait nécessaire, à côté d'une philosophie de la connaissance nourrie depuis Descartes, de développer une «théorie de l'action». Cette théorie ne peut aller sans une «théorie de la reconnaissance» ni une «vision de la reconnaissance de soi » qui remonte aux Grecs lorsqu'ils exprimaient leurs pensées sur l'agir et se présentaient comme redevables de leurs actes. Cette assurance relativement à sa «capabilité », cette reconnaissance de soi à l'actif ne peut aller sans son renversement au passif : «le soi demande à être reconnu». À la fois modalité de formation et d'évaluation, le mémoire doit permettre à un débutant, au moment où il entre dans l'action, de «se reconnaitre», de dire sa capacité à faire, sa croyance en son pouvoir d'agir, mais également d' «être reconnu» par des pairs expérimentés comme nouveau professionnel (Jorro, 2009).

Dans cette perspective, nous choisissons d'étudier, à titre exploratoire, et à grains fins, en contrepoint d'aspects souvent considérés comme insuffisants, quelques éléments d'un mémoire répondant aux attentes de l'institution à un double niveau, d'abord par les membres du jury chargé d'évaluer le mémoire et qui lui a attribué la meilleure mention, puis par la commission qui l'a jugé suffisamment exemplaire et modélisant pour le faire figurer sur le site de l'IUFM de l'académie de Lyon. Écrit par Aurélie Simonot ${ }^{2}$ en 2006, il s'intitule Effet de surprise et compréhension en lecture. Apprendre à lire, comprendre et interpréter des textes littéraires au cycle 2. Dans le cadre d'une formation par alternance, il s'inscrit dans la dernière année des mémoires écrits individuellement et encadrés dans des séminaires thématiques réunissant une quinzaine de stagiaires. Il se situe dans la mise en place de programmes promulgués en 2002, introduisant dès la maternelle l'initiation à la littérature.

Nous choisissons d'utiliser modestement les possibilités d'un prisme proposé dans Les puissances de l'expérience, par Ferry (1991) qui envisage une dynamique de construction des identités qui passe, à partir d'une «identité narrative» montrant le rôle fondateur de la narration comme premier mode de construction de l'identité, par une «identité

2. Je remercie Aurélie Simonot de m'avoir permis d'utiliser son travail et de mentionner son nom. Le mémoire est accessible sur le site de l'IUFM de Lyon et sur Internet à partir du nom de l'auteure. 
interprétative», puis une «identité argumentative», avant d'être parachevée par un quatrième mouvement appelé «reconstruction». Cette «identité reconstructrice», «en faisant redescendre la vérité sur terre» (p. 151), la soumet à son historicité radicale : «le discours reconstructif reconnait sa matière dans les autres discours, et l'histoire des autres est aussi sa propre histoire» (p. 154).

Nous utiliserons les outils de l'analyse des discours et de la linguistique énonciative et envisagerons le scripteur de ce mémoire comme un «être de discours» offrant différentes identités discursives observables dans un genre qui sera considéré comme «écrit de formation réflexif». Dans cet écrit, présentant une forte hétérogénéité discursive, l'auteur a, comme l'indique Guibert (2001, p. 181), à se situer entre différents «jeux de textes» notamment entre «infratexte», issu d'expériences vécues et «intertexte» provenant de recherches et discours scientifiques variés. Dans ces différents jeux de textes, il a également à articuler des savoirs hétérogènes qu'avec Vanhulle (2009b, p. 170) nous identifions de quatre types. Trois sont d'origine externe : «savoirs académiques» issus de la science, «savoirs institutionnels» répondant aux attentes de l'employeur et «savoirs de la pratique» venus du milieu professionnel. Ces savoirs sont à différencier d'un quatrième type qui représente des «savoirs expérientiels propres». Ainsi, entre implication et distance, le scripteur a à prendre différentes identités énonciatives parmi lesquelles nous distinguerons trois je essentiels : un «je autobiographique», avec un passé, des projets, des pratiques dont il a été l'acteur principal, un «je épistémique» qui, en référence à des écrits théoriques, analyse ses pratiques, et un «je communiquant» ayant à construire un lecteurinterlocuteur multiple, professionnel lui-même chargé de reconnaitre chez un néophyte des compétences professionnelles. Dans les cadres présentés par Ferry (1991), tous ces je sont mis en relation par un je épistémique qui applique, adapte, transpose des théories, dépasse l'identité expérientielle, justifie et argumente ses actions, théorise ce qui peut et doit être remis en circulation.

\section{Identités énonciatives en je, nous et on}

L'introduction, comme lieu stratégique d'ouverture et reflet de l'ensemble des positionnements énonciatifs du mémoire, nous a semblé un poste d'observation privilégié. À partir de l'évocation de l'histoire drôle qui fait rire tout le monde sauf une personne qui s'en trouve humiliée, cette introduction, par une série de retours en arrière successifs, 
remonte à un moment de formation où la conférencière affirme que c'est pour ceux qui ne comprennent pas les histoires drôles que l'enseignant travaille. Ce cours magistral appelle le souvenir d'une expérience vécue : "Il m'est souvent arrivé de faire semblant de rire dans une conversation alors que je n'en avais pas saisi tout l'enjeu et je trouve cela particulièrement frustrant.» $\left(\mathrm{M}^{3}\right.$, p. 2.) La remémoration de cette expérience intime permet elle-même de remonter au choix du sujet du mémoire et à la compréhension d'une motivation profonde jusque-là insue et subitement éclairée : «j'avais enfin la réponse à ma question : pourquoi est-ce que je tiens tant à faire travailler mes élèves sur des textes contenant une chute» (M, p. 2). L'image de la lumière surgit en hologramme du récit à chute : "J'aime ces moments d'éclairement quand tout à coup, à la fin, "on comprend", cette prise de recul en accéléré qui permet d'un coup de tout embrasser dans son ensemble et d'en apprécier les détails.» (M, p. 2.) Plus loin dans le mémoire, est convoquée une référence à Freud selon qui «tout phénomène n'apparait que dans un "après-coup", entre passé qui éclaire le présent et présent qui permet de "rétro-dire" le passé» (M, p. 11). Ce discours impliqué, cet auto-éclairement donne une première consistance à une identité professionnelle et à un rapport au savoir qui prend sens parce qu'il s'amarre au passé d'un je autobiographique «qui n'a pas à renier le sol natal de sa propre parole» (Flahault, 1978, p. 671). Utilisé cent-onze fois en trente-cinq pages, l'usage du je permet de faire du scripteur un auteur, créateur en propre de sa réflexion et d'un véritable travail sur soi qui est au cœur de ce que veut dire Ferry (1991).

L'anecdote de l'histoire drôle incomprise est relatée en on : «On peut toujours se faire répéter, se faire expliquer. Mais très vite on ne supporte pas de vivre en décalé, de comprendre par procuration.» (M, p. 2.) Cependant, dès la première phrase du mémoire, en tant que captatio benevolentiae à visée argumentative, l'expérience est prise en charge par un je «communiquant» qui, en nous, inclut le lecteur et fait appel à une expérience partagée : "Cette histoire, nous la connaissons tous $[\ldots] »(\mathrm{M}, \mathrm{p} .2)$. Ainsi se construit un ethos professionnel qui prédispose favorablement un jury face à un nouvel enseignant soucieux de faire réussir tous les élèves. Ce nous communiquant se retrouve tout au long du mémoire pour accompagner le lecteur en coénonciateur, parfois en alternance dans la même phrase avec un je/me acteur : "Ces questions nous le verrons plus tard me serviront autrement» (M, p. 21).

3. Toute citation extraite du mémoire sera référencée avec la lettre M. 
Ce nous équivalent à un je + vous fait place en fin d'introduction à un nous équivalent d'un je épistémique, «nous de majesté» ou «nous de modestie» habituel dans les écrits universitaires à la recherche d'un savoir : "Une définition des termes de la problématique [...] amènera à nous interroger sur la place d'un dispositif permettant l'entrainement à la compréhension. [...] Pour finir, nous nous attacherons à [...].» (M, p. 2)

Les guides et conseils aux jeunes chercheurs prescrivent habituellement un choix d'énonciation unique et proscrivent l'usage du je selon un lieu commun bien souvent contraire à ce qui s'observe dans la science où le je est régulièrement utilisé (Tutin, 2010). À l'opposé de ces prescriptions, on observe tout au long du mémoire un usage diversifié et équilibré selon les parties (voir annexe 1) de je, nous et on et parfois un entremêlement dans la même partie, le même paragraphe ou la même phrase de je et de nous. Ces emplois pourraient passer pour incompétence ou négligence si nous n'y reconnaissions un usage subtil de positionnements énonciatifs diversifiés à reconnaitre aux pratiquants ordinaires de la langue, inventifs et rusés, «producteurs méconnus et poètes de leurs affaires, inventeurs de sentiers dans les jungles de la rationalité fonctionnaliste» (de Certeau, 1990, p. 57).

\section{Le dialogue avec autrui}

Le je n'est pas un je solipsiste occupé de soi seul. À la différence de mémoires qui présentent parfois les stagiaires sur une ile déserte, sans collègues, ni formateurs, ni élèves (Guigue et Crinon, 2003, p. 113), «fantômes à peine évoqués dans un inventaire squelettique d'actes didactiques interchangeables» (Nonnon, 1995, p. 110), l'expérience est largement située : cadre géographique de l'école, milieu social des élèves, niveau scolaire individuel évalué à partir d'évaluations locales ou nationales, contexte institutionnel. Ces données feront de ce mémoire «un vestige archéologique» (Plane, 2008, p. 199) d'un temps où de nouveaux programmes ont laissé des enseignants titulaires démunis et transformé des stagiaires en explorateurs sans passé ni appui sur des savoirs pratiques circulant dans la profession: «Les conversations que j'ai eues avec l'enseignante titulaire m'ont permis de savoir que le travail sur la littérature de jeunesse exigé par les Nouveaux Programmes était encore peu présent dans l'établissement non faute de volonté mais de “savoir comment s'y prendre".» (M, p. 16) 
En l'absence de savoirs pratiques disponibles, quel rôle jouent les savoirs académiques? Associé à la production du savoir, le travail de références à des écrits théoriques fait partie des rituels langagiers des écrits de recherche et le recours à la citation s'avère indispensable : «Grande pourvoyeuse de noms propres, elle contribue à peupler le monde textuel» (Boch et Grossmann, 2001,p. 100). Les études sont nombreuses (Boch et Grossman [dir.], 2001; Rabatel, 2002; Kara, 2004), qui montrent les difficultés à citer le discours théorique et à tresser à son propre discours ce qui reste fondamentalement le discours d'autrui, d'autant plus étranger que souvent le scripteur le découvre en même temps que l'écriture du mémoire. En ce sens, nous avons tenté de retrouver les observables construits par Grossmann et al. (2009) qui ont cherché à voir comment se situe l'apprenti-chercheur par rapport au discours théorique. Or, nous ne trouvons pas, dans ce mémoire, le matériel lexical qui permet au jeune chercheur de se situer dans une communauté scientifique, dans une filiation par rapport à des prédécesseurs auxquels il fait allégeance, mais également dont il se démarque afin d'affirmer la possibilité d'une contribution nouvelle à la science.

En revanche, nous repérons, dans les références aux discours théoriques, un usage du syntagme prépositionnel selon ${ }_{E}$, employé seize fois, qui nous semble mériter attention. Souvent paraphrasable par pour ${ }_{E}$ ou d'après ${ }_{E}$ qui ne sont utilisés chacun qu'une ou deux fois, selon présente certaines nuances étudiées par Borillo (2004) et Coltier \& Dendale (2004) avec le pronom de première personne : selon moi donne un ton péremptoire, indique que le locuteur qui énonce $\mathrm{P}$ estime qu'il décrit le réel et se soumet à un ordre du monde, tend vers la vérité commune objective, donne l'image de celui qui sait, qui a soupesé ce qu'il dit. Pour moi s'associe à une vue de l'esprit, décrit une vision singulière du moi revendiquée comme telle puisque seul compte ce que croit le locuteur. Selon associé à des noms propres permet d'introduire des points de vue différents. Reprenant la «théorie des cadres de discours» de Charolles (1997), Schrepfer (2004) montre que selon ${ }_{E}$ a une portée textuelle et introduit un «univers énonciatif» qui renvoie en propre à la pensée d'autrui et n'engage que lui, souvent distingué dans un ilot citationnel guillemeté clairement borné et fermé par une référence bibliographique. Ainsi, dans ce mémoire, les dix-sept auteurs convoqués et cités quarante-sept fois (voir annexe 2) permettent d'importer des savoirs sur les processus en jeu dans la compréhension en lecture et d'introduire, à propos de la lecture littéraire, la notion de «texte résistant» mis en vogue par Tauveron (2002) au moment de la mise en place, 
à l'école primaire, de nouveaux programmes qui sont cités neuf fois. Ces différentes références inscrivent un stagiaire dans une communauté professionnelle où elles circulent et sont tenues pour les plus récentes et les plus valides du moment, faisant apparaitre des consensus mais aussi des dissensus entre discours des chercheurs et discours institutionnels.

Souvent les critiques signalent les citations comme simple exhibition de savoirs et d'auteurs servant de garants derrière lesquels s'abrite l'auteur citant. Dans sa théorie de l'argumentation, Angenot (2008) affirme que l'espoir raisonnable de convaincre son interlocuteur est généralement si «mince» ou si «nul» qu'essentiellement les humains « argumentent pour se justifier, pour se procurer face au monde une justification [...]. La conscience de soi se confond avec le désir de se justifier vis-à-vis d'un auditoire raisonnable» (p. 441). Cette dimension n'est pas absente de cet écrit qui, en tant qu'objet d'évaluation, s'inscrit dans une communication asymétrique d'évalué à évaluateur. Pourtant certaines configurations laissent paraitre une autre dimension montrant comment l'auteur intègre à son propre discours «ces voix du dehors» (Boch et Grossmann, 2001, p. 91).

$\mathrm{Au}$ fur et à mesure, les citations font appel à des auteurs qui se situent sur le terrain de la didactique dont les visées praxéologiques sont constitutives du champ. Les citations visent alors l'action et manifestent ainsi une visée fonctionnelle et «heuristique» qui n'est jamais signalée dans les guides méthodologiques (Boch et Grossmann, 2001, p. 94). Elles aident à imaginer des modalités de travail et à les justifier en leur donnant une certaine rationalité. Elles servent à vertébrer la réflexion didactique qui devient tremplin de l'action. Il n'est pas possible à ce moment de douter des savoirs sur lesquels le nouvel enseignant s'appuie sous peine de s'empêcher d'agir ou de surseoir aux décisions. Aussi trouve-t-on de façon récurrente une configuration particulière entre discours citant et discours cité où la référence entraine vers la décision qui fait passer de l'étude à l'action et transforme l'étudiant en enseignant, «centre de décision» (Ricœur, 2004, p. 123) responsable de ses choix et sujet comptable de ses actes. En voici un exemple :

Selon M. BUTLEN et M. COUET «La mise en réseau facilite la prise de conscience des procédures» $(2003$, p. 222). Je ne me contenterai donc pas d'une œuvre mais aborderai des textes de genres différents. Ces mêmes auteurs ajoutent «L'aptitude à mettre à distance les schèmes de lecture les plus classiques résulte d'une familiarisation et d'observations répétées» (2003, p. 230). Je fais donc le choix de l'entrainement afin d'installer une compétence et des références. (M, p. 18) 
Cette cotextualisation efficiente en Selon ${ }_{E}$ en position initiale, suivi de $J e+d o n c+$ verbe d'action, relie l'agir à des savoirs issus de la recherche, marque une appropriation subjective de savoirs et la quête de conduites rationalisées qui s'appuient sur des autorités. C'est dans la deuxième partie, consacrée aux choix didactiques effectués, que les marques de première personne sont les plus nombreuses, utilisées soixante-et-onze fois alors qu'elles ne le sont jamais en première partie et seulement treize fois en troisième partie. Ce processus de "privatisation» de l'univers des textes légitimes (Nonnon, 1995, p. 96) est le signe d' «une manipulation créatrice des connaissances» (Vanhulle, 2009a, p. 76), d'une co-construction de savoirs et de soi-même dans laquelle nous voyons une caractéristique spécifique d'un écrit servant à la construction et au développement d'une identité professionnelle propre à des enseignants débutants en formation.

\section{Écrire l'expérience}

À la difficulté de construire l'intertexte s'ajoute celle de construire l'infratexte qui se bâtit à partir du vécu, ce à quoi, comme l'indique Nonnon (1995, p. 95), les étudiants sont le moins habitués puisqu'ils ont, dans leur carrière scolaire et universitaire, surtout écrit à l'intérieur d'un univers sémiotique homogène, sur des textes et avec des textes. Écrire à partir d'un vécu extérieur au monde des textes et en faire un objet de discours affronte d'emblée le scripteur au problème de l'hétérogénéité. Les outils manquent pour mettre en scène le réel et construire une représentation des pratiques. Cela est d'autant plus difficile que le scripteur en est un acteur impliqué et qu'il s'aventure en terre inconnue :

Lors de mon tout premier contact avec les élèves je n'avais pas d'attente les concernant. En effet, ce stage était pour moi la première occasion de me confronter à de jeunes enfants en situation d'apprentissage et je n'avais, malgré mes lectures théoriques, aucune idée de ce que je pouvais attendre d'enfants de 6-7 ans en milieu scolaire. (M, p. 15)

Dire le réel est périlleux puisque ce qui est à dire c'est le poids du réel, le choc de l'épreuve des faits en décalage avec ce qui a été imaginé. Souvent prohibée au nom de la scientificité, la narration est souvent stigmatisée dans les mémoires. Le récit d'expérience a pourtant son intérêt. À cet égard, un passage du mémoire qui constitue une «scène» au sens narratologique du terme peut être interrogé. L'auteure a choisi de mettre le projecteur sur ce qu'on pourrait appeler un «moment de crise» où rien de ce qui a été anticipé ne s'est passé : 
Gwendoline la première annonce qu'elle a écrit : «Félicien est sur Mars». À ma grande surprise tout le monde est d'accord et acquiesce, $100 \%$ des élèves ont répondu «sur Mars» alors que je pensais que deux ou trois auraient découvert la chute. Cette unanimité empêche tout débat mais pas pour les raisons envisagées. Je demande à Gwendoline de justifier sa réponse et elle me répond : «c'est écrit : je vous écris de Mars.» Afin d'amorcer le débat j'annonce que pour ma part je n'y crois pas et leur demande de me convaincre avec les éléments du texte, ils ont des difficultés à se justifier et ont l'air de me trouver bizarre. (M, p. 22)

Dans l'urgence d'agir, sur le vif et dans l'instant, se reconnait une activité tacticienne, l'intelligence rusée que les Grecs appelaient mètis (Détienne et Vernant, 1974) qui fait utiliser les moyens du bord dans le danger, un art de faire du quotidien, «science du concret» du «bricoleur» qui élabore «des ensembles structurés non pas directement avec d'autres ensembles structurés, mais en utilisant des résidus et des débris d'évènements [...], des bribes et des morceaux, témoins fossiles de l'histoire d'un individu ou d'une société» (Lévi-Strauss, 1990, p. 36). C'est ce que fait la stagiaire en recyclant un questionnaire traditionnel mis au rebut au moment de la préparation puisqu'il avait été envisagé pour un scénario contraire où les élèves auraient trop vite compris la chute de la nouvelle :

Je vois alors l'intérêt des questions complémentaires que j'avais préparées et demande : [...]. Louis le premier annonce que «Félicien veut se venger» et finit par dire qu'il fait apporter le sandwich dans le grenier. Je relance : [...]. Les élèves ont des difficultés à enchainer leurs propos et reviennent souvent à «Eh bien moi je pense qu'il est sur Mars». Je les dirige alors de nouveau vers les pistes ouvertes par leur camarade mais nous les perdons, préoccupés qu'ils sont de répondre à la question initiale de la maitresse. (M, p. 22)

La scène est décisive car c'est à partir d'elle que se construit un savoir expérientiel à propos des conceptions de jeunes élèves qui considèrent que ce que dit le texte est vrai, et ne cessent de revenir à l'argument «c'est comme ça parce que c'est écrit» (M, p. 25). Ils se trouvent écartelés dans une double contrainte quand la maitresse, qui aussi dit le vrai, affirme le contraire, ce qui les fait la trouver «bizarre» et les désoriente. Le récit n'est pas un seul récit factuel. Le passage d'une «identité narrative» à une «identité interprétative» telle que la définit Ferry (1991) se marque par l'inclusion de boucles explicatives qu'indiquent les phrases et propositions mises en italique dans les deux pas- 
sages précédemment cités : la subordonnée introduite par alors que, les propositions coordonnées dont l'une commente l'autre et entre lesquelles et est équivalent de donc. La participiale en fin de paragraphe donne signification à un comportement d'élèves et sépare le nous du ils.

Il est intéressant de noter comment, dans cette dernière phrase, l'auteure se désigne sous trois formes différentes. Au je, protagoniste principal d'une scène de classe, succède un nous désignant maitre et élèves comme une communauté discursive dont les membres sont étroitement interdépendants et orientés vers un même but ${ }^{4}$. Puis le nous éclate en ils et la maitresse. L'expérience brise l'illusion de communauté discursive idéale et révèle que, même s'ils sont fortement liés, maitre et élèves sont aussi séparés et mus par des représentations et savoirs différents. Cet écart entre sphère du maitre et sphère des élèves fait découvrir au débutant cette «altérité fondamentale» à reconnaitre comme «fondement de la relation pédagogique» (Plane, 2004, p. 51). Ainsi la construction rétrospective d'un évènement didactique et pédagogique qui a mis momentanément en échec permet l'acquisition de savoirs expérientiels qui autorise un sujet engagé dans une recherche de rationalisation, à travers la verbalisation de l'expérience, à se reconnaitre comme professionnel en développement.

\section{Bilan d'un parcours de savoirs}

Un parcours de savoirs s'est inscrit dans un parcours de formation effectué dans un temps court : "Il y a plusieurs mois je me suis posé une question [...]. Aujourd'hui je connais [...]» (M, p. 35). Dans la conclusion, à travers une énonciation avec quinze marques de $1^{\text {re }}$ personne, les verbes et les caractérisations de procès intellectuels et réflexifs manifestent l'acquisition de savoirs professionnels : «j'identifie surtout $[\ldots] », \ll$ je connais mieux $[\ldots] », \ll$ je retiendrai $[\ldots] », \ll$ je sais quelles difficultés $[\ldots] »$, «J'ai pris conscience $[\ldots] »$, , «e travail de mise en place et d'amélioration du dispositif m'a ainsi permis tout à la

4. L'enseignant est si fortement investi et désireux d'enrôler ses élèves que parfois il s'inscrit lui-même dans le travail qu'il prescrit aux élèves : «Je leur ai aussi présenté le questionnaire comme moyen de vérifier eux-mêmes si le travail que nous allions faire ensemble les ferait évoluer, en indiquant que nous répondrions exactement au même questionnaire en fin de période.» (M, p. 14) 
fois d'élargir mes connaissances [...], de découvrir les outils [...] et de me les approprier» (M, p. 35). La formule métadiscursive souligne le rôle de l'écriture : «La rédaction de ce mémoire a été l'occasion de mener une réflexion sur $[\ldots] »(\mathrm{M}, \mathrm{p} .35)$.

Sur la voie d'une réflexivité croissante, le mémoire étudié offret-il le mouvement de «reconstruction» tel que le définit Ferry (1991)? Pour répondre à cette question, il nous semble intéressant d'observer le traitement accordé tout au long du mémoire à la notion de «texte résistant» qui sert de support à la séquence. Mise en circulation en 2002 par les travaux de Tauveron, la notion a fait fortune et s'est répandue rapidement au point de devenir un passage obligé et quasi officiel des discours sur l'enseignement de la littérature. À notre connaissance, dans le champ des écrits de la recherche en didactique, elle n'est remise en question dans sa définition et ses usages que depuis peu (Quet, 2009). La prise de conscience de l'historicité d'une notion prend du temps. Il faut du temps aussi pour en voir les effets dans des applications didactiques et pédagogiques ou dans des expérimentations de chercheurs qui ont contribué au succès de cette notion mais invitent désormais à la prudence et incitent à développer une attention vigilante à la réception de ce genre de texte par de très jeunes élèves (Quet, 2009, p. 107). À la fin du mémoire, la notion de «texte résistant» n'est pas réinterrogée, ni les conceptions de la lecture littéraire ou de l'apprentissage qu'elle masque. Pourtant, les effets d'un travail avec ce genre de texte sont soigneusement étudiés à travers les réactions des élèves puis à travers le dépouillement d'un questionnaire de début et de fin de séquence qui essaye de mesurer les évolutions : beaucoup d'élèves ont progressé et citent désormais la procédure de retour en arrière qui aide à mieux comprendre un texte. En revanche, ils se sentent moins capables de lire seuls un texte puisqu'ils pensent que, pour comprendre un texte, il faut demander à l'adulte, ce qui les rend plus dépendants de l'enseignante qui visait à les rendre plus autonomes. D'autres effets pervers sont mesurés qui montrent la restriction du fait littéraire si l'on s'en tient aux textes résistants et surtout l'effet négatif sur l'apprentissage de la lecture lui-même quand celui-ci s'appuie sur ces textes qui rusent et suscitent la défiance chez le lecteur, à l'inverse d'œuvres qui favorisent «une identification aux héros, ces héros, qui, par une lecture participative aident à l'entrée en littérature des lecteurs débutants » (M, p. 30). Les conclusions sont les mêmes que celles du chercheur.

Dans la troisième partie du mémoire, des sous-parties, intitulées «Questions en suspens» ou «Nouvelles perspectives», permettent un 
retour sur l'expérience et, en même temps, le retour aux auteurs cités en première partie. Une réévaluation de l'ensemble du dispositif montre de nouveaux savoirs sur des élèves de cycle 2 , leurs possibilités et leurs difficultés, des analyses reconsidérées des textes choisis, de nouveaux choix de textes plus adaptés avec «des outils d'aide que sait consulter un professionnel» (M, p. 35). L'emploi de on, utilisé trente fois au lieu de huit et trois fois en première et deuxième parties ainsi que l'usage plus fréquent de la modalité déontique manifestent des savoirs plus assurés, plus appropriés, plus incorporés, et surtout réévalués à l'aune d'un retour réflexif sur l'expérience qui s'apparente à ce que Ferry (1991) nomme « reconstruction».

Il nous semble, à travers un parcours singulier, avoir aperçu quelques spécificités de cet objet qu'on appelle «mémoire professionnel». En raison de ses finalités, qui ne sont pas indépendantes des cadres praxéologiques dans lesquels il se produit, le mémoire professionnel se distingue du mémoire de recherche. En tant qu' «écrit de formation réflexif», il manifeste une «grammaire» particulière, à reconnaitre autant par ceux qui l'évaluent que par celui qui l'écrit pour se reconnaitre et être reconnu comme nouveau professionnel en formation, entre deux statuts, celui d'étudiant et celui d'enseignant débutant.

\section{RÉFÉRENCES BIBLIOGRAPHIQUES}

Angenot M. (2008) : Dialogues de sourds. Traité de rhétorique antilogique, Paris, Mille et une nuits, coll. «Essais».

Boch F. et Grossmann F. (dir.) (2001) : Lidil, $\mathrm{n}^{\circ} 24$, Apprendre à citer le discours d'autrui.

Boch F. et Grossmann F. (2001) : «De l'usage des citations dans le discours théorique: des constats aux propositions didactiques», Lidil, $\mathrm{n}^{\circ} 24$, p. 91-112.

Borillo A. (2004) : «"Les adverbes d'opinion forte” selon moi, à mes yeux, à mon avis... point de vue subjectif et effet d'atténuation», Langue française, $\mathrm{n}^{\circ} 142$, juin 2004, p. 31-40.

Certeau M. de (1990) : L'invention du quotidien, t. 1 : Arts de faire, Paris, Gallimard, coll. «Folio essais ».

Charolles M. (1997) : «L'encadrement du discours, univers, champs, domaines et espaces », Cahier de recherche linguistique, ${ }^{\circ} 6$, URA 1035 LAnDisCo, université Nancy 2. 
Coltier D. et Dendale P. (2004) : «La modalisation du discours de soi : éléments de description sémantique des expressions pour moi, selon moi et à mon avis », Langue française, $\mathrm{n}^{\circ} 142$, juin 2004, p. 41-57.

CRINON J. (dir.) (2003) : Le mémoire professionnel des enseignants, observatoire des pratiques et levier pour la formation, Paris, L'Harmattan.

Cros F. (dir.) (1998) : Le mémoire professionnel en formation des enseignants, un processus de construction identitaire, Paris, L'Harmattan.

Détienne M. et Vernant J-P. (1974) : Les ruses de l'intelligence. La mètis des Grecs, Paris, Flammarion, coll. «Champs essais».

Flahaut F. (1978) : La parole intermédiaire, Paris, Seuil.

FERRY J-M. (1991) : Les puissances de l'expérience, t. 1 : Le sujet et le verbe, Paris, Cerf.

Garcia-Debanc C. (1994) : «Regards croisés sur une copie», Pratiques, $n^{\circ} 84$, p. 69-122.

Gomez F. (2001) : Le mémoire professionnel objet de recherche et outil de formation, Bruxelles, De Boeck Université.

GonNin-Bolo A. et Benoit J-P. (2004) : Le mémoire professionnel en IUFM. Bilan de recherches et questions vives, Paris, INRP.

Grossmann F., Tutin A. et Garcia Da Silva P-P. (2009) : «Filiation et transferts d'objets scientifiques dans les objets de recherche», Pratiques, $\mathrm{n}^{\circ} 143-144$, p. 187-202.

GuiberT R. (2001) : «Identité énonciative et système représentationnel de l'écrit : une notion pour construire la relation de soi à l'autre», Questions de recherche en éducation, $t .2$ : Action et identité, INRP, CRFCNAM Paris, p. 177-194.

Guigue M. et Crinon J. (2003) : «Les modalités de présence des élèves dans les mémoires professionnels», dans J. Crinon (dir.), Le mémoire professionnel des enseignants, observatoire des pratiques et levier pour la formation, Paris, L'Harmattan, p. 113-132.

JoRro A. (2009) : La reconnaissance professionnelle en éducation, Presses de l'université d'Ottawa.

KARA M. (2004) : «Pratiques de la citation dans les mémoires de maitrise», Pratiques, $\mathrm{n}^{\circ}$ 121-122, p. 111-142.

Lévi-Strauss Cl. (1990) : La pensée sauvage, Paris, Pocket, coll. «Agora».

Nonnon E. (1995) : «Les interactions lecture-écriture dans l'expérience d'une écriture professionnelle : le mémoire des professeurs débutants», Pratiques, $\mathrm{n}^{\circ}$ 86, p. 93-122.

Plane S. (2004) : «La rencontre des jeunes professeurs de français avec leurs classes. Découverte de l'altérité et tension pédagogique», Le français aujourd'hui, $\mathrm{n}^{\circ} 146$, p. 47-56.

- (2008) : «La place de l'écriture dans les mémoires des enseignants stagiaires français : un révélateur des tensions internes au processus de formation», Diptyque, n 13 , p. 191-222. 
Quet F. (2009) : «Qui résiste aux albums résistants ?», Diptyque, n 17, p. $97-115$.

RABATEl A. (2002) : «Le sous-énonciateur dans les montages citationnels», Enjeux, no 54, p. 52-67.

Riceur P. (2004) : Parcours de la reconnaissance, Paris, Gallimard, coll. «Folio essais ».

$\mathrm{S}_{\mathrm{CHÖN}} \mathrm{D}$. (1994) : Le praticien réflexif. À la recherche du savoir caché dans l'agir professionnel, Montréal, Logiques.

SCHREPFer G. (2004) : «Sur la portée textuelle des expressions introductrices de cadres de discours en selon $x$ : les indices de clôture des univers énonciatifs », dans R. Delamotte-Legrand (dir.), Les médiations langagières, vol. 1 : Des faits de langue aux discours, DYALANGCNRS, université de Rouen, p. 248-263.

Simonot A. (2006) : Effet de surprise et compréhension en lecture. Apprendre à lire, comprendre et interpréter des textes littéraires au cycle 2, IUFM de Lyon.

Tutin A. (2010) : «Lexique verbal et positionnement de l'auteur dans les articles en sciences humaines », Lidil, $\mathrm{n}^{\circ} 42$, p. 15-40.

Vanhulle S. (2009a) : Des savoirs en jeux au savoir en «je». Cheminements réflexifs et subjectivation des savoirs chez de jeunes enseignants en formation, Berne, Peter Lang.

- (2009b) : «Savoirs professionnels et construction sociodiscursive de l'agir», Bulletin suisse de linguistique appliquée, n 90, Pratiques langagières et formation professionnelle, L. Filllietaz (éd.), p. 167-188. 


\section{ANNEXES}

\section{Annexe 1 : je, nous, on, selon}

\begin{tabular}{|l|l|l|l|l|l|l|}
\hline & $\begin{array}{l}\text { total } \\
35 \mathrm{p} .\end{array}$ & $\begin{array}{l}\text { introduction } \\
2 \mathrm{p} .\end{array}$ & $\begin{array}{l}1^{\text {re }} \text { partie } \\
9 \mathrm{p} .\end{array}$ & $\begin{array}{l}2^{\mathrm{e}} \text { partie } \\
11 \mathrm{p} .\end{array}$ & $\begin{array}{l}3^{\mathrm{e}} \text { partie } \\
11 \mathrm{p} .\end{array}$ & $\begin{array}{l}\text { conclusion } \\
1 \mathrm{p} .\end{array}$ \\
\hline je & 65 & 8 & & 45 & 4 & 8 \\
\hline mon, mes, ma & 20 & 2 & & 13 & 3 & 2 \\
\hline me, $\mathrm{m}^{\prime}$, moi & 26 & 2 & & 13 & 5 & 1 \\
\hline total $1^{\text {re }}$ pers. & 111 & 12 & & 71 & 13 & 15 \\
\hline nous, nos & 26 & 3 & 1 & 8 & 7 & 7 \\
\hline on & 56 & 8 & 15 & 3 & 30 & \\
\hline selon $\mathrm{x}$ & 16 & 1 & 9 & 4 & 2 & \\
\hline
\end{tabular}

\section{Annexe 2 : citations}

\begin{tabular}{|c|c|c|c|c|c|c|}
\hline Noms & total & introduction & $1^{\mathrm{re}}$ partie & $2^{\mathrm{e}}$ partie & $3^{\mathrm{e}}$ partie & conclusion \\
\hline $\begin{array}{l}\text { Cèbe } \\
\text { Cébe, Goigoux }\end{array}$ & 2 & 1 & & & 1 & \\
\hline Tauveron & 16 & & 12 & 1 & 3 & \\
\hline $\begin{array}{l}\text { Grossmann, } \\
\text { Tauveron }\end{array}$ & 2 & & & 2 & & \\
\hline Giasson & 11 & & 9 & 1 & 1 & \\
\hline IO & 9 & & 5 & 3 & 1 & \\
\hline ONL & 1 & & & & 1 & \\
\hline Picard & 3 & & 1 & & 2 & \\
\hline Eco & 2 & & 2 & & & \\
\hline Iser & 1 & & 1 & & & \\
\hline Freud & 1 & & 1 & & & \\
\hline Butlen, Couet & 3 & & 1 & & 2 & \\
\hline Beltrami, Quet & 2 & & & 1 & 1 & \\
\hline Chartier & 1 & & 1 & & & \\
\hline Faivre-Mercier & 1 & & 1 & & & \\
\hline Poslaniec & 1 & & & 1 & & \\
\hline Calame-Gippet & 1 & & & 1 & & \\
\hline 17 auteurs + IO & 57 & 1 & 37 & 8 & 19 & \\
\hline
\end{tabular}

